\title{
The influence of zero value subtraction on the performance of a new laser fluorescence device for approximal caries detection
}

\author{
Jonas de Almeida Rodrigues • Isabel Hug • Adrian Lussi
}

Received: 20 October 2007 / Accepted: 24 January 2008 / Published online: 29 February 2008

(C) Springer-Verlag London Limited 2008

\begin{abstract}
The aim of this study was to assess the influence of the zero value subtraction on the performance of laser fluorescence (LFpen) for approximal caries detection. Three areas (cuspal, middle and cervical) of both mesial and distal buccal surfaces of 78 permanent molars were assessed using both wedge-shaped (WDG) and tapered wedgeshaped (TWDG) tips. With the addition of the average, one cut-off value for each area was obtained and the performance was assessed. The areas under the receiver operating characteristics (ROC) curve, specificity, sensitivity and accuracy with and without the zero value subtraction were calculated. The McNemar test revealed a statistically significant difference for specificity at thresholds $\mathrm{D}_{1}, \mathrm{D}_{2}$ and $\mathrm{D}_{3}$ (WDG) and $\mathrm{D}_{1}$ and $\mathrm{D}_{2}$ (TWDG) when the zero value subtraction was not performed. Influence of the zero value subtraction on the LFpen performance was observed for approximal caries detection. However, when modified cut-off values were used, the zero value subtraction could be eliminated.
\end{abstract}

Keywords Approximal caries · DIAGNOdent .

DIAGNOdent pen $\cdot$ Laser fluorescence $\cdot$ Zero value

\section{Introduction}

The phenomenon of fluorescence can be observed in the dental tissues and is based on the principle that carious dental tissues emit higher fluorescence than sound tissues

J. de Almeida Rodrigues $(\bowtie) \cdot$ I. Hug $\cdot$ A. Lussi

Department of Preventive Restorative and Pediatric Dentistry,

School of Dental Medicine, University of Bern,

Freiburgstrasse 7,

CH 3010 Bern, Switzerland

e-mail: jorodrigues@hotmail.com do when stimulated by pulsed red light. The laser fluorescence (LF) device has shown good results in caries detection and has been recommended as a tool in the diagnosis process $[1,2]$.

The first LF device (DIAGNOdent, KaVo, Biberach, Germany) was developed in order to support the dentist in the diagnostic process, but it has been demonstrated that plaque, calculus, toothpaste, dental materials, stains, hypomineralized non-carious teeth or incorrect calibration show false-positive results [3-6]. Recently, a new laser fluorescence device (LFpen; DIAGNOdent pen, KaVo) was developed to be used on approximal and occlusal surfaces. This new device emits red light with a wavelength of 655 $\mathrm{nm}$ and includes a filter that blocks wavelengths below $655 \mathrm{~nm}$, which eliminates reflected and ambient light. A photodetector quantifies the fluorescent light passing through the filter and shows, in a digital display, a real time ("moment") and a maximum ("peak") value. Inside the solid fibre tip the excitation and fluorescence follows the same optic path of propagation in the opposite direction [7, 8], which is different from the first LF device [9].

According to the manufacturer's instructions, the sites should be calibrated against a ceramic reference (standard calibration) and afterwards against a sound spot on every tooth so that the zero value of fluorescence can be obtained. This step has not been followed by practising dentists, and there is no mention in the literature about the correct area in which the zero value should be recorded when the first LF device is being used. Some researchers only mentioned that the buccal surface was used but did not specify the area or did not perform the calibration [10-12].

In this context, it is important to highlight that the cut-off values for the LFpen were based on a zero value taken on the cuspal area of the buccal surface [8]. It can be assumed that the incorrect measurement, or the lack of it, might 


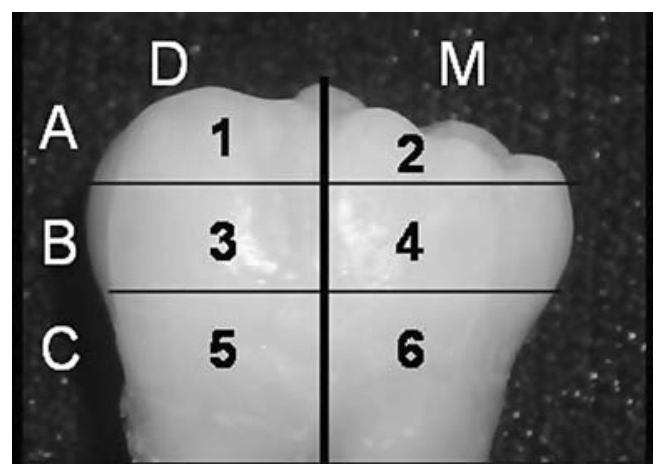

Fig. 1 Six sites on the buccal surface measured with the LFpen

result in a false assessment of the caries state and, hence, influence the treatment decision. Therefore, it was hypothesized that this second step of measurement could be eliminated, if new modified cut-off values were used. This would simplify the clinical procedures. For this reason, in our study, one data set was used to measure buccal surfaces and to determine different cut-off limits, while another was employed to assess the influence of these cut-off values on LF performance.

Thus, the aim of this in vitro study was to assess the influence of the zero value subtraction on the performance of the LFpen device for approximal caries detection.

\section{Materials and methods}

Seventy-eight extracted permanent human molars, frozen at $-20^{\circ} \mathrm{C}$ until required, were selected. All teeth had been extracted by dental practitioners in Switzerland (no water fluoridation, 250 p.p.m. $\mathrm{F}^{-}$in table salt). Prior to the extraction, the patients were informed about the use of the teeth for research purposes and their consent was obtained. Calculus and debris were removed, and the teeth were cleaned for $15 \mathrm{~s}$ with water and then for $10 \mathrm{~s}$ with Prophyflex with sodium bicarbonate (KaVo). Before being measured, they were defrosted for $3 \mathrm{~h}$. During the assessments they were stored in $100 \%$ humidity. The buccal surfaces of the selected teeth were divided in six different non-caries areas (sites 1 to 6; Fig. 1): three mesial (M) sites and three distal (D) sites, which were named cuspal (A), middle (B) and cervical (C) areas.

The mode of function of the LFpen device has been described in detail previously [8]: a laser diode serves as the light source. The first path is made so that the light from the solid fibre is transmitted to a large number of $40 \mu \mathrm{m}$ single fibres. The second path transfers the fluorescence to the photodetector. Separation of the fluorescence light from the excitation and reflected light is accomplished with a fluorescence filter with specific characteristics. The whole process is controlled by a microcontroller with connection to a speaker and a digital display showing the real time and peak value.

After standard calibration in a ceramic reference, we assessed each tooth with the LFpen, using for approximal caries detection two different sapphire fibre tips with the following dimensions: wedge-shaped (WDG; width $1.1 \mathrm{~mm}$ and thickness $0.4 \mathrm{~mm}$ ) and tapered wedge-shaped (TWDG; width $0.7 \mathrm{~mm}$ and thickness $0.4 \mathrm{~mm}$ ).

\section{Statistical analysis}

We performed statistical analysis considering each area $(M$, $\mathrm{D}, \mathrm{A}, \mathrm{B}$ and C) and each tip separately. As the paired $t$-test did not show statistically significant differences between the mesial and distal areas, the LF values of each area were combined. The normal distribution of the values was confirmed by a Q-Q plot, and the $t$-test [ $95 \%$ confidence interval (CI), at $P<0.05]$ was used (SPSS 14.0, Chicago, Ill., USA). As the values of the LF pen are discrete, we used a rounded average (rav) of each area, as well as among all the areas, for both tips, to define the new cut-off values, and the performance of the LFpen was assessed with data from a previous study [8]. We assessed LF performance by comparing the same data set without subtracting the zero value of fluorescence to the results, using the cuspal area (area A) for the zero value subtraction [8]. The sensitivity and specificity were obtained from the receiver operating characteristic (ROC) curve. This analysis involves a plot of pairs of sensitivity (true positive rate) and '100-specificity' (false positive rate) for a given cut-off value of a test, and the values are independent of the prevalence of the disease. The use of a gold standard is a prerequisite for the assessment of the ROC curve. The areas under the ROC curve for both tips were calculated and compared for each threshold as described earlier [13]. The values of sensitivity, specificity and accuracy were obtained for each area and for the three areas together. The comparison among the

Table 1 Average, standard deviations and frequencies of LFpen readings for both tips in cuspal (A), middle (B), cervical (C), mesial (M) and distal (D) areas

\begin{tabular}{lllll}
\hline Tip & Area & Average & SD & Frequencies (\%) \\
\hline WDG & A & 4.1 & 1.6 & $3.0-4.5(53.8)$ \\
& B & 6.1 & 3.4 & $5.0-6.5(34.7)$ \\
& C & 7.2 & 3.4 & $7.0-9.5(38.4)$ \\
& M & 5.8 & 2.4 & $2.0-8.0(88.3)$ \\
TWDG & D & 5.7 & 3.0 & $1.0-8.0(81.4)$ \\
& A & 4.7 & 1.7 & $3.0-6.0(73.1)$ \\
& B & 6.9 & 3.5 & $5.0-7.5(41.1)$ \\
& C & 8.3 & 5.4 & $8.0-9.5(17.9)$ \\
& M & 6.7 & 2.9 & $1.3-9.0(81.8)$ \\
& D & 6.5 & 3.6 & $1.0-8.0(77.3)$ \\
\hline
\end{tabular}


Table 2 Cut-off values of the LFpen for approximal caries detection taking into account the rounded average addition of zero value to the original cut-off limits from Lussi et al. [8] for each area (rav rounded average)

\begin{tabular}{|c|c|c|c|c|c|c|c|c|c|c|}
\hline \multirow[t]{2}{*}{ Histology } & \multicolumn{5}{|l|}{ WDG } & \multicolumn{5}{|l|}{ TWDG } \\
\hline & Original & A rav $=4$ & B rav $=6$ & $\mathrm{C}$ rav $=7$ & $\mathrm{ABC}$ rav $=6$ & Original & A rav $=5$ & B rav $=7$ & $\mathrm{C}$ rav $=8$ & $\mathrm{ABC}$ rav $=7$ \\
\hline $\mathrm{D}_{0}$ & $0-6$ & $0-10$ & $0-12$ & $0-13$ & $0-12$ & $0-9$ & $0-14$ & $0-16$ & $0-17$ & $0-16$ \\
\hline $\mathrm{D}_{1}$ & $6.1-9$ & $10.1-13$ & $12.1-15$ & $13.1-16$ & $12.1-15$ & $9.1-13$ & $14.1-18$ & $16.1-20$ & $17.1-21$ & $16.1-20$ \\
\hline $\mathrm{D}_{2}$ & $9.1-15$ & $13.1-19$ & $15.1-21$ & $16.1-22$ & $15.1-21$ & $13.1-22$ & $18.1-27$ & $20.1-29$ & $21.1-30$ & $20.1-29$ \\
\hline $\mathrm{D}_{3,4}$ & $>15$ & $>19$ & $>21$ & $>22$ & $>21$ & $>22$ & $>27$ & $>29$ & $>30$ & $>29$ \\
\hline
\end{tabular}

specificity, sensitivity and accuracy values was performed by the McNemar test (5\% significance level).

\section{Results}

The intervals with highest frequencies of LFpen readings with the average and standard deviation (SD) are listed in Table 1 . The paired $t$-test showed a statistically significant difference $(P<0.05)$ between the areas $\mathrm{A}, \mathrm{B}$ and $\mathrm{C}$ but no difference between $\mathrm{M}$ and $\mathrm{D}$. Furthermore, a statistical difference also was found when the two tips used in the same area were compared.

The calculated cut-off limits are shown in Table 2, as well as the related thresholds: $D_{0}$ was caries free, $D_{1}$ had caries extending up to halfway through the enamel, $\mathrm{D}_{2}$ showed caries in the inner half of the enamel, $\mathrm{D}_{3}$ had caries in the dentine and $\mathrm{D}_{4}$ indicated deep dentinal caries.

No statistically significant difference in the areas under the ROC curves with and without the zero value subtraction (Fig. 2 and Table 3) for the TWDG and WDG tips was found $(P>0.05)$. The values of sensitivity, specificity and accuracy calculated with the LF values from Lussi et al. [8] and the suggested limits are shown in Table 4, together with the same values when the original cut-off limits [8] were used without the zero value subtraction. The McNemar test revealed a statistically significant difference for specificity at thresholds $\mathrm{D}_{1}, \mathrm{D}_{2}$ and $\mathrm{D}_{3}$ (WDG) and $\mathrm{D}_{1}$ and $\mathrm{D}_{2}$ (TWDG) when the zero value subtraction was not performed. For the accuracy, this statistical difference was found at $\mathrm{D}_{1}$ and $\mathrm{D}_{3}$ for the WDG tip (Table 4).

\section{Discussion}

LF is aimed at the detection of caries lesions when hard dental tissues are stimulated by wavelengths of $655 \mathrm{~nm}$. Recent studies have shown that oral bacterial metabolites (porphyrins) may contribute to this fluorescence [14]. Thus,
Fig. 2 ROC curves for WDG and TWDG tips at different thresholds $\left(\mathrm{D}_{1}, \mathrm{D}_{2}\right.$ and $\left.\mathrm{D}_{3}\right)$. Continuous line without subtraction of the zero value, dashed lines with subtraction of the zero value. The approximal LF values used were taken from Lussi et al [8]
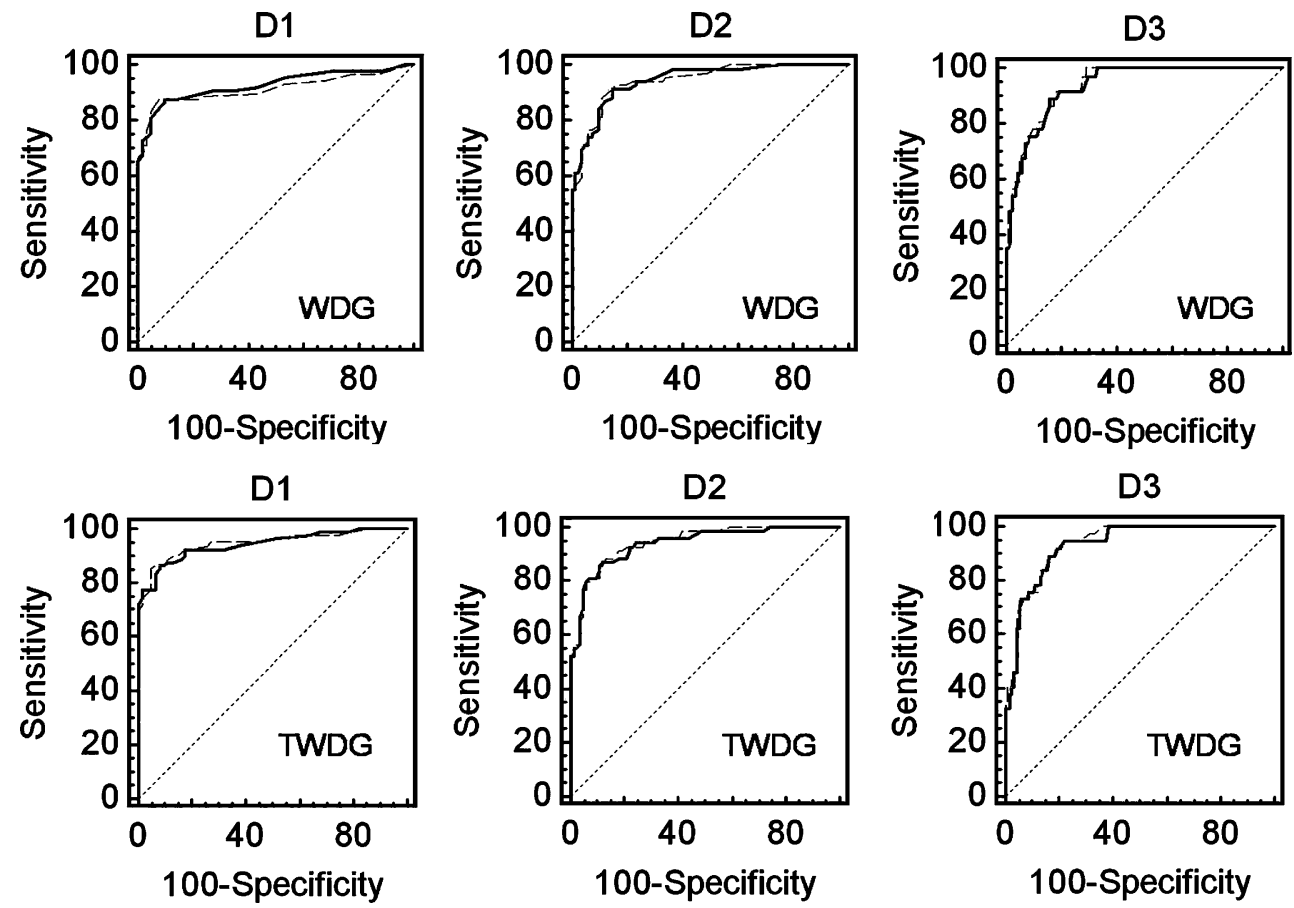
Table 3 Areas under the ROC curve, differences and $P$ values for WDG and TWDG tips at different thresholds. The approximal LF values used were taken from Lussi et al [8]

\begin{tabular}{llrr}
\hline Parameter & & & Area \\
& & $\mathrm{D}_{1}$ & $\mathrm{D}_{2}$ \\
\hline Without zero value subtraction & WDG & 0.927 & 0.945 \\
With zero value subtraction & WDG & 0.915 & 0.944 \\
& Difference & 0.013 & 0.001 \\
& P & 0.242 & 0.918 \\
Without zero value subtraction & TWDG & 0.943 & 0.938 \\
With zero value subtraction & TWDG & 0.949 & 0.943 \\
& Difference & 0.006 & 0.005 \\
& $P$ & 0.492 & 0.963 \\
& & & 0.477 \\
\hline
\end{tabular}

this method has been a valuable addition to conventional methods of caries detection [9, 10].

The new LFpen, like the first system, must be calibrated prior to use. In accordance with the manufacturer's instructions, the device is first calibrated against a ceramic reference, the fluorescence of which is known. After the standard calibration, the fluorescence value of a sound spot must be recorded (zero value) and subtracted later from the values assessed on the tooth surface. This is a somewhat time-consuming procedure, which is not well followed in practice. It is important to highlight that some variation in these measurements may change the detection performance and, consequently, the treatment decision. Some authors studying the first device and assessing the buccal surface found values varying by up to 6 units over the four quadrants [2], while others did not find differences in LF readings created by an absence of zero value subtraction [15].
In our study we assessed six different sites, using two tips of the new LF pen, but three areas were compared because no statistical difference was found between the mesial and distal sites. The frequencies showed that lower values of LF were found in cuspal (A) areas, increasing in the middle (B) and cervical (C) areas, with statistically significant difference. The highest values of fluorescence in cervical areas can be explained by the mineralization levels observed in the different areas of the teeth [16] and by the thinness of the enamel towards the cement-enamel junction, which allows high fluorescence measurements of the underlying dentine [17]. However, when mesial (M) and distal (D) areas were evaluated, the values showed no statistically significant difference, because the average among $\mathrm{A}, \mathrm{B}$ and $\mathrm{C}$ of each surface represents similar levels of mineralization in both surfaces.

The tested tips showed statistically different measurements $(P<0.05)$, probably because they have different

Table 4 Performance of the LFpen in detecting approximal caries using different cut-offs (taking into account the values showed in Table 2) at different thresholds. The approximal LF values used were taken from Lussi et al [8]

\begin{tabular}{|c|c|c|c|c|c|c|c|c|c|c|c|}
\hline \multirow[t]{2}{*}{ Parameter } & & \multirow[b]{2}{*}{ Cut-off } & \multicolumn{3}{|c|}{ Specificity } & \multicolumn{3}{|c|}{ Sensitivity } & \multicolumn{3}{|c|}{ Accuracy } \\
\hline & & & $\mathrm{D}_{1}$ & $\mathrm{D}_{2}$ & $\mathrm{D}_{3}$ & $\mathrm{D}_{1}$ & $\mathrm{D}_{2}$ & $\mathrm{D}_{3}$ & $\mathrm{D}_{1}$ & $\mathrm{D}_{2}$ & $\mathrm{D}_{3}$ \\
\hline \multirow[t]{6}{*}{ WDG } & \multirow[t]{5}{*}{ Without zero value subtraction } & A & 0.90 & 0.88 & 0.82 & 0.86 & 0.87 & 0.89 & 0.88 & 0.88 & 0.84 \\
\hline & & $\mathrm{B}$ & 0.95 & 0.90 & 0.85 & 0.79 & 0.78 & 0.84 & 0.86 & 0.85 & 0.85 \\
\hline & & $\mathrm{C}$ & 0.92 & 0.90 & 0.85 & 0.80 & 0.84 & 0.86 & 0.85 & 0.87 & 0.85 \\
\hline & & $\mathrm{ABC}$ & 0.95 & 0.90 & 0.85 & 0.79 & 0.78 & 0.84 & 0.89 & 0.85 & 0.85 \\
\hline & & Original & $0.56^{*}$ & $0.94 *$ & $0.75 *$ & 0.92 & 0.77 & 0.92 & $0.77 *$ & 0.86 & $0.79 *$ \\
\hline & With zero value subtraction & Original & 0.92 & 0.89 & 0.82 & 0.88 & 0.87 & 0.89 & 0.90 & 0.88 & 0.84 \\
\hline \multirow[t]{6}{*}{ TWDG } & \multirow[t]{5}{*}{ Without zero value subtraction } & A & 0.93 & 0.89 & 0.82 & 0.83 & 0.84 & 0.89 & 0.87 & 0.87 & 0.84 \\
\hline & & $\mathrm{B}$ & 0.94 & 0.92 & 0.84 & 0.78 & 0.81 & 0.84 & 0.85 & 0.87 & 0.84 \\
\hline & & $\mathrm{C}$ & 0.98 & 0.93 & 0.86 & 0.77 & 0.81 & 0.84 & 0.86 & 0.88 & 0.86 \\
\hline & & $\mathrm{ABC}$ & 0.94 & 0.92 & 0.84 & 0.78 & 0.81 & 0.84 & 0.85 & 0.87 & 0.84 \\
\hline & & Original & $0.82 *$ & $0.78 *$ & 0.76 & 0.92 & 0.91 & 0.95 & 0.88 & 0.84 & 0.81 \\
\hline & With zero value subtraction & Original & 0.93 & 0.89 & 0.81 & 0.87 & 0.84 & 0.92 & 0.89 & 0.87 & 0.84 \\
\hline
\end{tabular}

$\mathrm{D}_{1}: \mathrm{D}_{0}=$ sound; $\mathrm{D}_{1}-\mathrm{D}_{3}=$ decayed.

$\mathrm{D}_{2}: \mathrm{D}_{0} \mathrm{D}_{1}=$ sound; $\mathrm{D}_{2} \mathrm{D}_{3}=$ decayed.

$\mathrm{D}_{3}: \mathrm{D}_{0}-\mathrm{D}_{2}=$ sound; $\mathrm{D}_{3}=$ decayed

*Statically significant difference within the same column (McNemar test, $P<0.05$ ) 
widths $(1.1 \mathrm{~mm}$ and $0.7 \mathrm{~mm})$, which is in accordance with the findings of an earlier study [8]. They are made of a solid single sapphire fibre, and the end of the tip has a prismatic shape that deflects the beam of excitation and collects it laterally along the longitudinal axis of the tip. However, this difference does not influence the performance of both tips when they are used in the clinical practice. Furthermore, cut-offs can be defined for each tip which can be used in clinical practice. For approximal caries detection, the tips should allow access to the approximal contact because of their thickness of $0.4 \mathrm{~mm}$ diameter. However, tight approximal contact could be a problem for caries detection [8]. The TWDG was an experimental tip developed to make penetration into the approximal space easier because of its smaller width.

The different storing methods could explain the different cut-off limits for the LF devices found in the literature, when in vitro studies were performed [7-9, 18, 19]. Previous studies in our laboratory showed that the fluorescence and, hence, the cut-off values decreased when teeth were stored in thymol, chloramine or formalin, and remained stable when teeth were stored frozen and only defrosted for measurement [20].

In a comparison of the areas under the ROC curve (with and without the zero value subtraction), no difference was found $(P>0.05)$, since this kind of analysis considered several cut-off limits, not taking a stipulated value [3]. Values higher than 0.9 were obtained, showing excellent accuracy of the tested method. Furthermore, the closer the ROC plot is to the upper left corner, the higher the overall accuracy of the test.

The influence of the different areas of the buccal surface on the LF readings could be confirmed. However, influence on the readings does not necessarily mean influence on the LF performance when one is attempting to detect approximal caries lesion. Therefore, we calculated different cut-off values, considering each area of the buccal surface for the zero value assessment and testing in order to eliminate the zero value subtraction. For this purpose, we used the laser fluorescence values from another sample [8] to assess the LFpen performance-supposing that the zero value subtraction was not performed in association with the use of new cut-off values.

Influence was observed when the specificities for WDG (at $\mathrm{D}_{1}, \mathrm{D}_{2}$ and $\mathrm{D}_{3}$ ) and TWDG tips (at $\mathrm{D}_{1}$ and $\mathrm{D}_{2}$ ) were analysed. Without zero value subtraction, the specificity at $\mathrm{D}_{3}$ was 0.75 for the WDG tip. This means that $25 \%$ of $\mathrm{D}_{3}$ lesions would be misclassified and probably treated operatively by mistake if the zero value subtraction was eliminated and no modified cut-off would be used. Statistically significant difference at $\mathrm{D}_{1}$ and $\mathrm{D}_{3}$ was found when the accuracy values were compared. In the face of these results, it could be suggested that the modified cut- offs which showed the best values of accuracy at $D_{1}$ and $D_{2}$ (cut-off $\mathrm{A}$, which we obtained taking into account area $\mathrm{A}$ for the zero value subtraction) should be used. This means that, if these cut-off values for the LF pen were used for approximal caries detection, the recording of the zero value would not be necessary anymore and the zero value subtraction could be eliminated. This study disagrees with that of Braga et al. [15], insofar as they found a different zero value of fluorescence measuring the centre of each tooth's buccal surface but no influence on LF performance in primary teeth without using a modified cut-off. Some authors studying the same subject in permanent teeth obtained other results and observed that the zero value subtraction influences the LF readings and indicates that this procedure should be performed before the diagnostic measurement [2]. In contrast to our study, the last two studies used the first LF device for occlusal caries detection, and no new cut-off limits were suggested.

It is important to point out that these results represent an in vitro situation, and caution must be taken when the cutoffs are used for clinical assessments. However, the setup of the study was such that no change due to storage in LF values should be expected [20]. Furthermore, those limits are not fixed, and measurements with the new LFpen should be considered as a second opinion only [8].

This is highly relevant, considering that the zero value assessment consumes time in clinical practice, where procedures should be simplified as much as possible, particularly when children are being treated [15]. Further in vivo studies using deciduous and permanent teeth should be made. It has to be kept in mind that the treatment decision depends on other patient variables, such as dietary and tooth brushing habits, caries activity and use of fluoride.

It can be concluded that the zero value subtraction influences the LFpen performance. However, this step could be eliminated when modified cut-off values are used.

\section{References}

1. Bader JD, Shugars DA (2004) A systematic review of the performance of a laser fluorescence device for detecting caries. J Am Dent Assoc 135:1413-1426

2. Braun A, Krause F, Jepsen S (2005) The influence of the calibration mode of a laser fluorescence device on caries detection. Caries Res 39:144-149

3. Mendes FM, Hissadomi M, Imparato JCP (2004) Effects of drying time and the presence of plaque on the in vitro performance of laser fluorescence in occlusal caries of primary teeth. Caries Res 38:104-108

4. Sheehy EC, Brailsford SR, Kidd EA, Beighton D, Zoitopoulos L (2001) Comparison between visual examination and a laser fluorescence system for in vivo diagnosis of occlusal caries. Caries Res 35:421-426 
5. Lussi A, Reich E (2005) The influence of toothpastes and prophylaxis pastes on fluorescence measurements for caries detection. Eur J Oral Sci 113:141-144

6. Rodrigues JA, Vita TM, Cordeiro RCL (2008) In vitro evaluation of the influence of aluminum oxide air abrasion of pits and fissures on detection of occlusal caries lesions in primary teeth. Ped Dent (in press).

7. Lussi A, Hellwig E (2006) Performance of a new laser fluorescence device for the detection of occlusal caries in vitro. J Dent 34:467-471

8. Lussi A, Hack A, Hug I, Heckenberg H, Megert B, Stich H (2006) Detection of approximal caries with a new laser fluorescence device. Caries Res 40:97-103

9. Lussi A, Imwinkelried S, Pitts NB, Longbotton C, Reich E (1999) Performance and reproducibility of a laser fluorescence system for detection of occlusal caries in vitro. Caries Res 33:261-266

10. Bamzahim M, Alijehani A, Shi XQ (2005) Clinical performance of DIAGNOdent in the detection of secondary carious lesions. Acta Odontol Scand 63:26-30

11. Alijehani A, Bamzahim M, Yousif MA, Shi XQ (2006) In vivo reliability of an infrared fluorescence method for quantification of carious lesions in orthodontic patients. Oral Health Prev Dent $4: 145-150$

12. Mendes FM, Oliveira E, Faria DLA, Nicolau J (2006) Ability of laser fluorescence device associated with fluorescent dyes in detecting and quantifying early smooth surface caries lesions. J Biomed Opt 11:0240071-00240076
13. Hanley JA, McNeil BJ (1983) A method of comparing the areas under receiver operating characteristic curves derived from the same cases. Radiology 148:839-843

14. Hibst R, Paulus R, Lussi A (2001) A detection of occlusal caries by laser fluorescence: basic and clinical investigations. Med Laser Appl 16:295-213

15. Braga MM, Mendes FM, Imparato JCP, Martins CR (2006) Effect of the calibration method of a laser fluorescence device for detecting occlusal caries in primary molars. Ped Dent 28:451454

16. Kodaka T, Debari K, Kuroiwa M (1991) Mineral content of the innermost enamel in erupted human teeth. J Electron Microsc 40:19-23

17. Krause F, Braun A, Eberhard J, Jepsen S (2007) Laser fluorescence measurements compared to electrical resistance of residual dentine in excavated cavities in vitro. Caries Res 41:135140

18. Shi XQ, Welander U, Angmar-Mansson B (2000) Occlusal caries detection with KaVo DIAGNOdent and radiography: an in vitro comparison. Caries Res 34:151-158

19. Pereira AC, Verdonschot EH, Huysmans MC (2001) Caries detection methods: can they aid decision making for invasive sealant treatment? Caries Res 35:83-89

20. Francescut P, Zimmerli B, Lussi A (2006) Influence of different storage methods on laser fluorescence values: a two-year study. Caries Res 40:181-185 